\section{Effectiveness and safety of flexible therapeutic schemes including first- and second- generation basal insulins during a pediatric summer diabetes camp}

\author{
Stefano Tumini, ${ }^{1}$ Laura Comegna, ${ }^{1}$ \\ Elisabetta Fioretti, ${ }^{1}$ Paola Guidone, ${ }^{1}$ \\ Gabriella Levantini, ${ }^{1}$ Daniele Panichi, ${ }^{1}$ \\ Milena Catenaro, ${ }^{1}$ Ilaria Rossi, ${ }^{1}$ \\ Flavia Amaro, ${ }^{1}$ Giusi Graziano, ${ }^{2}$ \\ Maria Chiara Rossi, ${ }^{2}$ Paola Cipriano ${ }^{1}$
} 1Department of Pediatrics University of Chieti; ${ }^{2}$ CORESEARCH - Center for Outcomes Research and Clinical Epidemiology, Pescara, Italy

\section{Abstract}

Outcomes of insulin analogues in pediatric diabetes camps are poorly investigated; no data is available about insulin degludec (IDeg).Our aim was to assess impact of insulin therapy adopted by the participants to a 4-day diabetes camp held in 2017, hypothesizing a possible excess risk of hypoglycemia in patients treated with IDeg. Overall, 40 children with type 1 diabetes (mean age $13.4 \pm 3.0$ years; $62.5 \%$ males) attended the camp $(20.0 \%$ on continuous subcutaneous insulin infusion and $80.0 \%$ on multiple daily injections - MDI). Among children in MDI regimen, $71.9 \%$ were treated with IDeg as basal insulin and $28.1 \%$ with glargine U100 (IGlar). All patients used Lispro or Aspart as short-acting insulin. Daily plan of the camp included educational sessions, physical exercise, 3 main meals and 2 snacks. At the arrival, IGlar and short-acting insulin doses were revised according to existing guidelines, while IDeg dose was revised based on an empirical individualized approach. At the arrival, insulin doses were reduced in 22 participants $(-19.4 \pm 10.5 \%)$, while doses were increased in 17 children $(+17.8 \pm 12.7 \%)$, based on individual needs. No statistically significant between-group difference emerged in mean blood glucose and glucose variability. No excess risk of hypoglycemia was found in the IDeg group. The study suggests similar effectiveness and safety of different insulin schemes when associated with appropriate diabetes education and management, and flexible dose adjustments. Despite its longer halflife and the lack of a validated algorithm, IDeg was not associated with an excess risk of hypoglycemia.

\section{Introduction}

Pediatric diabetes camps are part of global management of type 1 diabetes (T1DM). ${ }^{1}$ They offer the opportunity to children and their parents to share experiences and acquire knowledge and practical skills for home diabetes care, with a positive impact on psychological well-being.2,3

Comprehensive guidelines for the management of T1DM during diabetes camps have been developed. ${ }^{4}$ Sport is a key component of camps; therefore, blood glucose levels and insulin therapy require a strict management to ensure an adequate response to physical exercise while avoiding hypoglycemia. ${ }^{5}$ In addition, camps represent controlled places in which to begin or consolidate physical activities; sport is a milestone in the treatment of T1DM and it should be performed not only at school, but also as an integral part of a global healthy lifestyle. ${ }^{6}$

Risk of late hypoglycemia in this population persists for at least 24 hours after exercise; this phenomenon is due to the increase in insulin sensitivity and the increase in circulating levels of insulin, exposing the children to a significant risk of nocturnal hypoglycemia.6,7

In previous studies, several insulin schemes have been assessed in patients participating in diabetes camps. ${ }^{7-9}$ Before the availability of insulin analogues, these studies took into consideration human insulins in patients treated with twice daily or multiple daily insulin injections (MDI), fixed doses of premix insulins, combinations of isophane or basal insulin and regular or short-acting analogues prior to the availability of long-acting insulin analogues. 7,8 In other studies, continuous subcutaneous insulin infusion (CSII), or MDI schemes including intermediate / long-acting insulin (insulin glargine, insulin detemir or NPH) were adopted. 9

However, evidence on risk of hypoglycemia is poor, especially for pediatric patients in MDI; furthermore, no data is available about the use of insulin degludec (IDeg) in diabetes camps. This issue is particularly relevant, since the preventive reduction of IDeg insulin before physical activity is not feasible, due the peculiar pharmacokinetic properties of IDeg (24hour half-life and steady-state of blood concentration after 4 days from start of therapy). 10,11

This real-life study had the aim of assessing effectiveness and safety of the different insulin schemes adopted by the participants (CSII or MDI including first or second generation of basal analogues plus
Correspondence: Stefano Tumini, Department of Pediatrics, University of Chieti,Via dei Vestini,66100 Chieti, Italy.

Tel.: +0871358014

E-mail: stefano.tumini@gmail.com

Key words: Type 1 diabetes, pediatric diabetes, diabetes camp, degludec, glargine, insulin analogues, continuous subcutaneous insulin infusion.

Acknowledgements: The authors thank the parents and children who participated in this research, as well as the nurses, and staff members. The authors are very grateful to the association "ABCDEF" (Associazione Bambini Con Diabete e Famiglie) local Association of parents of diabetic children for help in camp organization.

Contributions: ST, conception and design, data interpretation, drafting the manuscript, guarantor of accuracy and integrity of the manuscript. LC, EF, PG, GL, DP, MC, IR, FA, PC, acquisition of data, critical revision and approval of accuracy and integrity of the manuscript. GG, data analysis. MCR data analysis and drafting the manuscript.

Conflict of interest: S.T. has participated in advisory boards for Novo Nordisk, Sanofi Aventis, Eli Lilly and Lifescan. He has also received speaker honoraria from Roche Diagnostics, Novo Nordisk, Sanofi Aventis and Harmonium Pharma. The remaining authors have no disclosure. The editorial assistance was provided by Airon Communication through a Novo Nordisk S.p.A. unconditional grant. The authors of the publication are fully responsible for the contents and conclusions. Novo Nordisk S.p.A. did not influence and has not been involved in the data interpretation and statistical analysis presented in the manuscript

Funding: Diabetes camp supported by the local patients' association "ABCDEF" (Associazione Bambini Con Diabete e Famiglie). Editorial assistance provided by Airon Communication through a Novo Nordisk S.p.A. unconditional grant.

Availability of data and materials: Study database not publicly available. Data available upon reasonable request to the authors.

Ethics approval and consent to participate: The study protocol was approved by the local Ethics Committee.

Informed consent: Informed consent was signed by parents of all patients included in the analysis.

Received for publication: 25 July 2019. Accepted for publication: 28 February 2020.

This work is licensed under a Creative Commons Attribution NonCommercial 4.0 License (CC BY-NC 4.0).

(C) Copyright: the Author(s), 2020

Licensee PAGEPress, Italy

Pediatric Reports 2020; 12:8254

doi:10.4081/pr.2020.8254 
short-acting insulin), hypothesizing a possible excess risk of hypoglycemia in patients treated with IDeg. Outcomes of T1DM management during the 4-day camp were blood glucose levels, glycemic variability, and hypoglycemic episodes.

\section{Materials and Methods}

This was an observational longitudinal prospective study. Data on all children participating to the diabetes camp held on 4 - 9 August 2017 in Fara Filiorum Petri, Abruzzo, Italy was collected.

Children were eligible for the camp if they had had a diagnosis of T1DM (ISPAD criteria) at least 1 year before, were treated with any insulin scheme (CSII or MDI), and had already received carbohydrate counting and T1DM self-management education. Parents participated in the camp only if children were less than 7 years old.

Daily plan included educational sessions, physical exercise of medium-high intensity, and 3 main meals and 2 snacks.

Educational and physical activities were organized taking into consideration three age groups: 7-10, 11-13, and 14-18 years.

One hour in the morning and one hour in the afternoon were devoted to sport (swimming in the morning and soccer, volleyball, basket, or judo in the afternoon). Children were involved in other activities during the remaining time (play, dance, etc..). Physical activities were executed in the presence of expert trainers and healthcare operators.

In accordance with current guidelines to prevent hypoglycemia, in patients treated with insulin glargine (IGlar) a $0-20 \%$ reduction of the dose was evaluated at the arrival day. Reduction entity was established on an individual basis, considering age, insulin needs, pubertal stage, frequency of hypoglycaemic episodes, sport attitudes, and intensity, duration and type of physical activity expected in the camp. A similar approach was not allowed in patients treated with insulin IDeg, since a dose reduction would have been requested from 3-4 days before the camp based on the pharmacokinetics, but no validated clinical recommendations exist on how to minimize risk of hyperglycemia and need for bolus corrections. ${ }^{4-6}$ On the other hand, data on adults document that in patients treated with IDeg performing low, moderate, and high intensity exercise, IDeg dose can be maintained stable while short-acting insulin dose is reduced proportionally to the patient needs. ${ }^{12,13}$ However, when deemed appropriate by the diabetologist, IDeg doses could be modified on the day of arrival according to the level of training, the level of glycemic control at home, and the individual risk of hypoglycaemia of the child.

In patients treated with CSII, basal infusion dose was revised at the arrival day on an individual basis following the current clinical recommendations and taking into consideration age, BMI, insulin daily dose, diabetes remission, pubertal status, and physical activity. Patients treated with CSII used the short-acting insulin analogue; they followed an algorithm suggesting a basal dose reduction 60-90 minutes before exercise and were instructed to disconnect the pump whenever necessary. 5

All patients (MDI and CSII) were trained to reduce pre-prandial doses before exercise on an individual basis or to perform an extra intake of carbohydrates (CHO). Dose reduction considered patient age, metabolic control, pubertal phase, insulin requirement, type of basal insulin (IGlar vs. IDeg vs. CSII), and glycemic trend in the previous days.

All patients in charge of the diabetes center promoting the camp had already received standard education on $\mathrm{CHO}$ counting and the correction of pre-prandial hyperglycemia using the insulin-carbohydrates (I:CHO) ratio and the insulin sensitivity factor (ISF); these latter are routinely assessed to adjust insulin therapy in these patients. ${ }^{14}$ During the camp, from the day 2 to the day 4, blood glucose monitoring, insulin administration, calculation of doses based on the I:CHO ratio, and daily and corrective insulin doses were supervised by diabetes specialists. ${ }^{3,4}$

A patient diary was filled in by each participant to report blood glucose levels, insulin bolus and basal insulin doses, ketonemia (if blood glucose $\geq 250 \mathrm{mg} / \mathrm{dl}$ ), $\mathrm{CHO}$ intake of each meal, I:CHO ratio, and ISF.

Blood glucose levels were checked before and 2 hours after each meal and in the presence of hypoglycemia symptoms. The last blood glucose measurement before night rest was performed at 11.00 - 12:00 pm; nightime blood glucose levels were checked by professional nurses between 01:00 and 03:00 am. If values $\leq 70 \mathrm{mg} / \mathrm{dl}$ were found, sugary liquids were administered (about 9 grams for a weight of $30 \mathrm{~kg}$ or 15 grams for a weight of $50 \mathrm{~kg}$ corresponding to about $0.3 \mathrm{~g}$ of glucose $/ \mathrm{kg}$ ), and blood sugar levels were measured at intervals of 10-15 minutes until the target blood glucose values were restored. 15

The study protocol was approved by the local Ethics Committee and the informed consent was signed for all patients included in the analysis.

\section{Statistical analyses}

Baseline variables included age, sex, pubertal status, duration of diabetes, body weight, body mass index (BMI), honeymoon phase, insulin regimen, $\mathrm{HbA}_{1} \mathrm{c}$, I:CHO ratio, physical activity.

Follow-up variables (i.e. study endpoints) included fasting blood glucose values (measured at 8 a.m.), daily blood glucose levels, blood glucose variability, and hypoglycemic episodes (i.e. values of selfmonitoring blood glucose $\leq 70 \mathrm{mg} / \mathrm{dl}$ and $<$ $50 \mathrm{mg} / \mathrm{dl}$, total, daytime and nocturnal).

Self-monitoring blood glucose measurements were downloaded on the physician computers using the DiasendTM system. Data relative from the 7 days before and the 7 days after the camp was extracted.

Baseline characteristics were expressed as mean and standard deviation or percentage for continuous and categorical variables, respectively. They were compared by insulin regimen using non-parametric Wilcoxon test and Fisher's exact test, as appropriate.

Mean glycemic levels were computed by day by insulin regimen.

Glucose variability was expressed as Coefficient of Variation (CV). ${ }^{16}$ It was computed for each patient, and average values by day were evaluated. The formula used for $\mathrm{CV}$ computation is:

$$
\mathrm{CV}(\%)=100 \times \frac{\mathrm{SD}(\text { daily glycemia })}{\text { Mean (daily glycemia) }}
$$

Daytime range included all values measured from 7:00 a.m. to 11:00 p.m.; nocturnal range included all values measured from 0:00 a.m. to 6:00 a.m.

Longitudinal linear models for repeated measures were applied to assess trends over time in continuous endpoints. All longitudinal models took into consideration four time points, i.e. day 1 , day 2 , day 3 , and day 4. An unstructured correlation type was used to account for within-patient correlation over time. Results were expressed as estimated mean and estimated mean change from baseline with their $95 \%$ confidence intervals (CIs). P-values $<0.05$ were considered statistically significant.

Number and percentage of patients with at least 1 hypoglycemic episode and incidence rates of hypoglycemic episodes were assessed by insulin regimen through Poisson regression. Results are expressed as Incidence Rates and 95\%Confidence Intervals.

All analyses were performed using SAS software release 9.4 (SAS Institute, Cary, NC, USA). 
with CSII $(+51.5 \pm 48.1 \%), 8$ were treated with IDeg $(+35.1 \pm 28.9 \%)$, and 1 with IGlar $(+18.1 \%)$. Pre-prandial insulin doses were adjusted based on the planned amount of $\mathrm{CHO}$ in the meals and physical activity. The first day of camp pre-prandial doses were reduced in 22 children $(-27.9 \pm 14.4 \%)$ of whom 5 treated with CSII $(-29.3 \pm 19.1 \%)$, 12 treated with IDeg $(-29.36 \pm 14.3)$, and 5 treated with IGlar $(-23.0 \pm 14.4 \%)$. Pre-pranand MDI patients for pre-prandial boluses used short-acting analogues Lispro (25.0\%) or Aspart (75.0\%). Basal insulin was prevalently administered before lunch, but it was administered before breakfast and before dinner in over $40 \%$ of patients treated with IDeg and IGlar.

Baseline characteristics overall and by insulin regimen are reported in Table 1 . No statistically significant differences among the three groups emerged in terms of average HbA1c levels; $32.5 \%$ of participants had $\mathrm{HbAlc} \leq 58 \mathrm{mmol} / \mathrm{mol}(7.5 \%)$ at the camp arrival. Two patients in the IGlar group were in honeymoon phase. Regular physical activity was performed by a significantly higher proportion of participants in the IDeg group (65.2\%), as compared to CSII (37.5\%) and IGlar (11.1\%) groups.

\section{Dose adjustment at arrival day}

At arrival day, basal insulin was reduced in 18 participants $(-22.3 \pm 14.5 \%)$, of whom 5 were treated with CSII ($22.7 \pm 18.1 \%), 7$ were treated with IDeg, ($24.3 \pm 15.4 \%)$, and 6 were treated with IGlar $(-19.3 \pm 12,4 \%)$. Basal dose was increased in 12 participants, of whom 3 were treated estimated means and standard error. dial insulin doses were increased in 16 participants $(+33.2 \pm 24.9 \%)$, of whom 3 treated with CSII $(+52.3 \pm 34.0 \%), 11$ treated with IDeg $(+32.8 \pm 21.0 \%)$, and 2 treated with IGlar $(+6.5 \pm 1.0 \%)$. Pre-prandial doses were left unmodified in the two children in honeymoon phase. Total insulin doses were reduced in 22 participants $(-19.4 \pm 10.5 \%)$, while doses were increased in 17 children $(+17.8 \pm 12.7 \%)$.

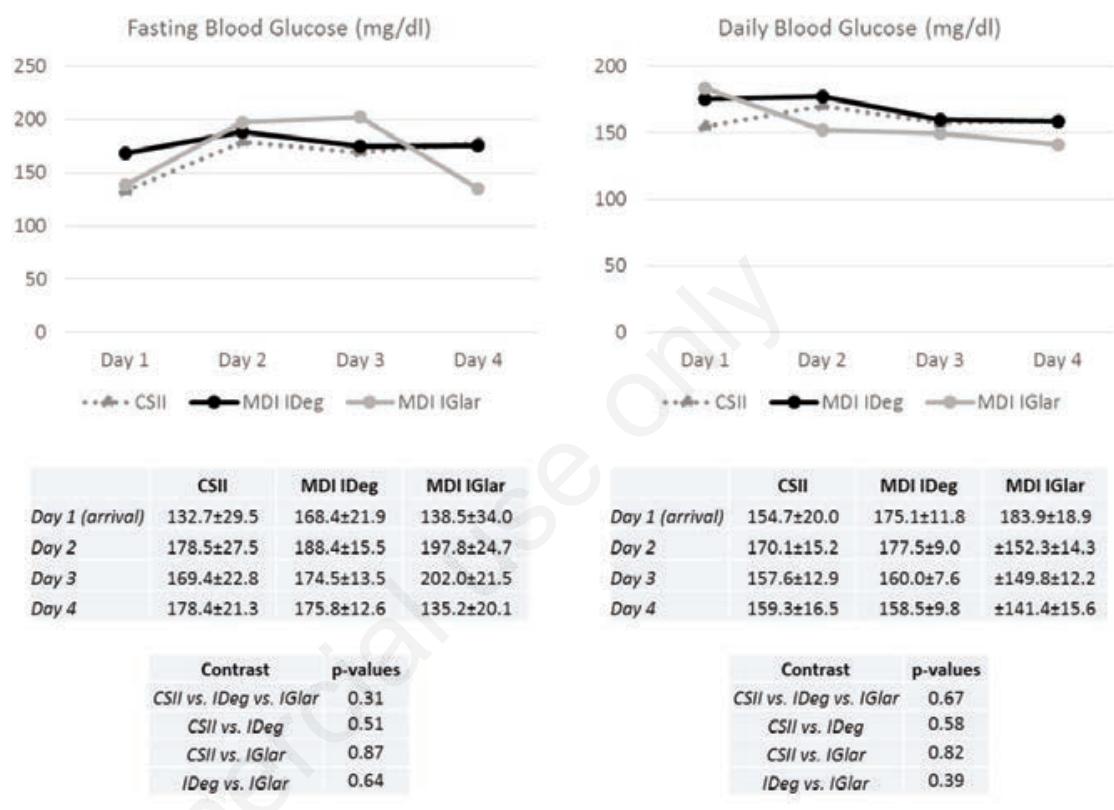

Figure 1. Trends by day and by insulin regimen of blood glucose levels. Data expressed as

Table 1. Baseline characteristics.

\begin{tabular}{|c|c|c|c|c|c|}
\hline Variable & Overall & CSII & MDI (IDeg) & MDI (IGlar) & P-value \\
\hline No. & 40 & 8 & 23 & 9 & \\
\hline Males (\%) & 62.5 & 37.5 & 60.9 & 88.9 & 0.10 \\
\hline Age (years) & $13.4 \pm 3.0$ & $14.5 \pm 4.8$ & $13.4 \pm 2.1$ & $12.2 \pm 2.8$ & 0.29 \\
\hline Pubertal (\%) & 75.0 & 60.0 & 86.9 & 55.5 & 0.10 \\
\hline Weight (Kg) & $53.4 \pm 13.7$ & $53.5 \pm 15.3$ & $54.4 \pm 12.7$ & $50.5 \pm 16.0$ & 0.78 \\
\hline BMI (Kg/m2) & $21.6 \pm 3.6$ & $22.1 \pm 3.4$ & $21.7 \pm 3.4$ & $20.6 \pm 4.3$ & 0.67 \\
\hline $\mathrm{HbAlc}(\mathrm{mmol} / \mathrm{mol})$ & $60.6 \pm 10.6$ & $59.9 \pm 8.1$ & $61.1 \pm 10.0$ & $60.1 \pm 14.8$ & 0.94 \\
\hline Regular sport activity (\%) & 47.5 & 37.5 & 65.2 & 11.1 & 0.02 \\
\hline Honeymoon phase (\%) & 5.0 & 0 & 0 & 22.2 & 0.08 \\
\hline Basal insulin dose (UI) & $23.3 \pm 11.3$ & $19.0 \pm 8.9^{*}$ & $25.8 \pm 9.6$ & $21.1 \pm 15.9$ & 0.29 \\
\hline Pre-prandial insulin dose (UI) & $24.3 \pm 12.3$ & $18.8 \pm 5.9$ & $25.9 \pm 11.6$ & $24.9 \pm 17.3$ & 0.38 \\
\hline Basal/Bolus Ratio & $1.1 \pm 0.4$ & $1.2 \pm 0.6$ & $1.1 \pm 0.4$ & $1.0 \pm 0.3$ & 0.60 \\
\hline Insulin sensitivity factor & $66.6 \pm 10.6$ & $59.9 \pm 26.0$ & $58.1 \pm 27.2$ & $91.7 \pm 56.1$ & 0.06 \\
\hline Insulin: CHO ratio (g per $1 \mathrm{UI}$ ) & $12.7 \pm 8.7$ & $18.0 \pm 13.5$ & $10.4 \pm 5.2$ & $13.9 \pm 9.4$ & 0.09 \\
\hline Short acting insulin (\%) & & & & & 0.24 \\
\hline Aspart & 55.0 & 75.0 & 43.5 & 66.7 & \\
\hline Lispro & 45.0 & 25.0 & 56.552 & 33.3 & \\
\hline
\end{tabular}

*Basal insulin infusion of short-acting insulin. 


\section{Follow-up}

On average, $6.9 \pm 1.2$ self-monitoring blood glucose measurements/day were performed during the camp. Mean fasting blood glucose and mean daily blood glucose levels are reported in Figure 1. No statistically significant between-group differences emerged.

CV levels during the 4-day camp and in the 7 days before and after the camp are reported in Figure 2. No statistically significant differences emerged among groups.

Overall, 109 episodes of hypoglycemia $\leq 70 \mathrm{mg} / \mathrm{dl}$ and 23 episodes of hypoglycemia $<50 \mathrm{mg} / \mathrm{dl}$ occurred. An increasing incidence of hypoglycemia during the camp in the three groups was documented, but no statistically significant betweengroup difference emerged (Figure 3). No episode of severe hypoglycemia with impaired cognitive abilities, seizure or coma or need for assistance from another person and / or administration of glucagon occurred.

Changes in insulin pro-kg requirements before and during the camp are reported in Table 2. During the camp, the I:CHO ratio increased from day 2 to day $4: 11.2 \pm 6.1 v s$. $14.4 \pm 7.7 \mathrm{~g} \times 1 \mathrm{UI}(\mathrm{p}<0.001)$, while intake of carbohydrates increased by $15.8 \%$ (day 2 vs. $4: 218.0 \pm 55.9$ vs. $252.5 \pm 39.7 \mathrm{~g}$, $\mathrm{p}<0.001)$.

\section{Discussion}

\section{Main findings}

To the best of our knowledge, this is the first study evaluating the impact of IDeg in a pediatric diabetes camp.

Fasting blood glucose levels were more stable in IDeg group than in the CSII and IGlar group, although no statistically significant difference emerged. No excess risk of hypoglycemia was found in IDeg group.

The first day of the camp, total insulin doses were decreased by about $20 \%$ in $55 \%$ of the children, while they were increased by about $18 \%$ in $42.5 \%$ of the children.

Incidence of hypos increased during four days in all groups, because of insulin sensitivity increase, without suggesting an excess risk in the IDeg group in spite of the absence of an algorithm for a specific dose adjustment. The risk was minimized through a bolus adjustment driven by $\mathrm{CHO}$ counting and ISF.

\section{Comparisons with existing knowledge}

Only a few studies have investigated the management of insulin doses during diabetes camps, the largest part conducted before first- and second-generation basal insulin analogues were made available. Data from real world experiences are scant and based on heterogeneous populations.79,17 Many recommendations are thus based on data deriving from experimental studies involving adult populations or expert opinions, suggesting a dose reduction between $10 \%$ and $40 \%$ of the dose administered at home. ${ }^{18-21}$

This is the first study involving children treated with short-acting and long-acting

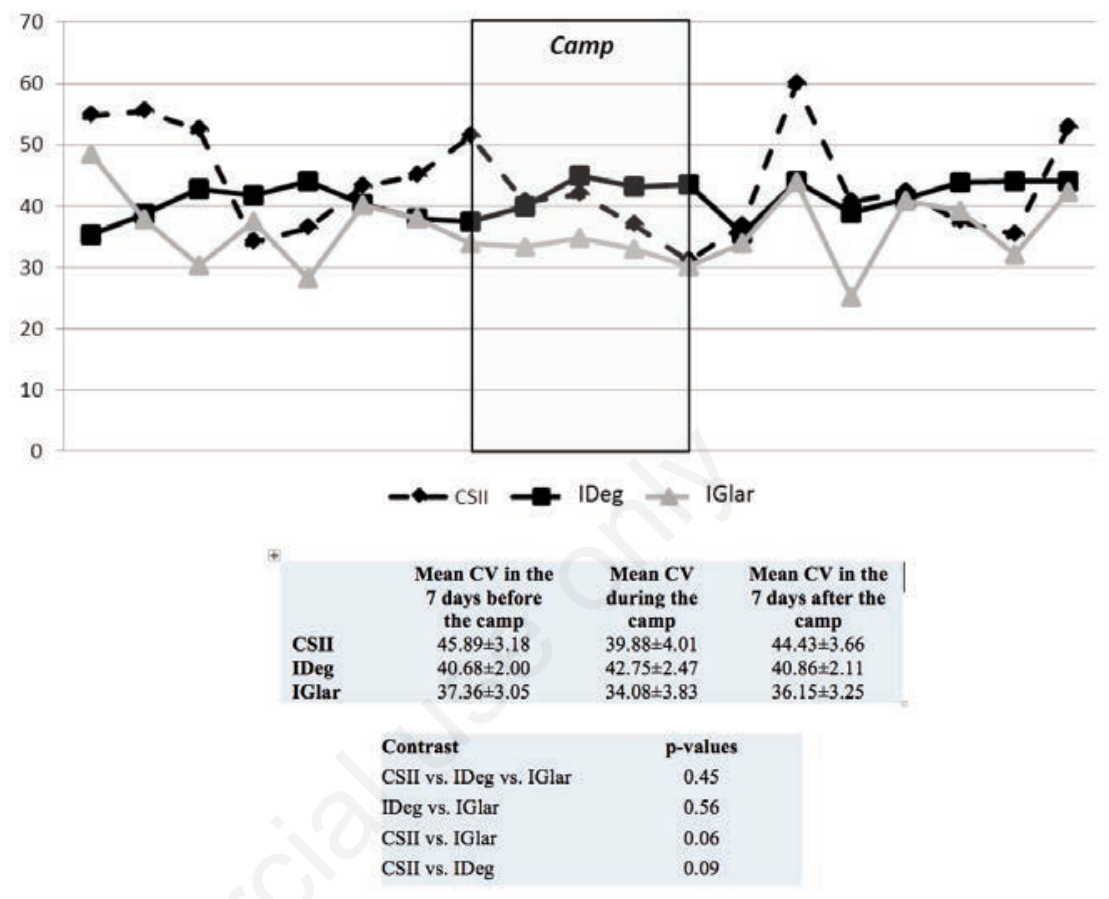

Figure 2. Trends of coefficient of variation of blood glucose during the camp and in the seven days before and after the camp.

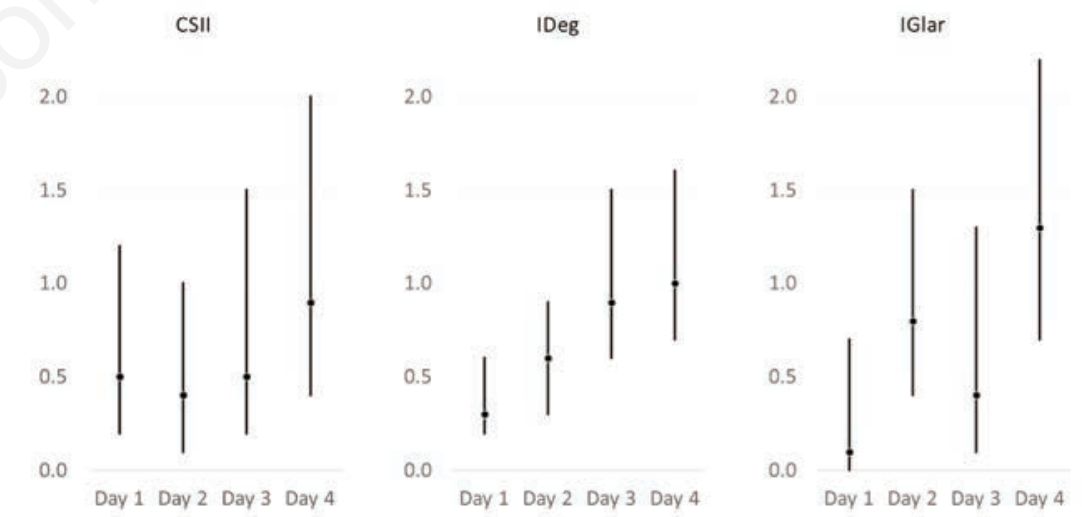

Data are expressed as episodes per person/day and $95 \%$ confidence intervals. No statistically significant benween-group differences emerged.

$\begin{array}{lcccc} & \text { CSII } & \begin{array}{c}\text { MDI } \\ \text { (IDeg) }\end{array} & \begin{array}{c}\text { MDI } \\ \text { (IGlar) }\end{array} & \text { p-value } \\ \text { N } & 8 & 23 & 9 & \\ \text { Total hypo }<=70 \mathrm{mg} / \mathrm{dl} & 87.5 & 100 & 77.8 & 0.06 \\ \text { Total hypo }<50 \mathrm{mg} / \mathrm{dl} & 50.0 & 43.5 & 44.4 & 0.94 \\ \text { Daytime hypo }<=70 \mathrm{mg} / \mathrm{dl} & 87.5 & 95.6 & 77.8 & 0.06 \\ \text { Daytime hypo }<50 \mathrm{mg} / \mathrm{dl} & 50.0 & 34.8 & 44.4 & 0.78 \\ \text { Nocturnal hypo }<=70 \mathrm{mg} / \mathrm{dl} & 12.5 & 34.8 & 11.1 & 0.89 \\ \text { Nocturnal hypo }<50 \mathrm{mg} / \mathrm{dl} & 0 & 8.7 & 0 & 1.00\end{array}$

Figure 3. Hypoglycemic events during the diabetes camp by insulin regimen. A) Incidence of hypo by day during the camp. B) $\%$ of patients with at least 1 hypoglycemic episode during the camp. 
Table 2. Change in insulin pro-Kg requirement before and during the camp.

\begin{tabular}{llccccc} 
Variable & & All & CSII & IDeg & IGlar & P-value \\
\multirow{2}{*}{ Pre camp } & Basal insulin & $0.44 \pm 0.15$ & $0.38 \pm 0.13$ & $0.48 \pm 0.12$ & $0.42 \pm 0.21$ & 0.25 \\
& Preprandial insulin & $0.45 \pm 0.17$ & $0.38 \pm 0.19$ & $0.47 \pm 0.16$ & $0.44 \pm 0.20$ & 0.49 \\
& Total insulin requirement & $0.89 \pm 0.28$ & $0.76 \pm 0.24$ & $0.95 \pm 0.24$ & $0.87 \pm 0.38$ & 0.28 \\
Day 2 & Basal insulin & $0.43 \pm 0.17$ & $0.34 \pm 0.12$ & $0.49 \pm 0.15$ & $0.38 \pm 0.20$ & 0.05 \\
& Preprandial insulin & $0.45 \pm 0.19$ & $0.34 \pm 0.12$ & $0.49 \pm 0.17$ & $0.44 \pm 0.24$ & 0.14 \\
& Total insulin requirement & $0.88 \pm 0.28$ & $0.68 \pm 0.12$ & $0.98 \pm 0.24$ & $0.82 \pm 0.39$ & 0.06 \\
\hline \multirow{2}{*}{ Day 4 } & Basal insulin & $0.44 \pm 0.16$ & $0.36 \pm 0.09$ & $0.49 \pm 0.15$ & $0.38 \pm 0.20$ & 0.06 \\
& Preprandial insulin & $0.41 \pm 0.15$ & $0.33 \pm 0.10$ & $0.44 \pm 0.15$ & $0.38 \pm 0.16$ & 0.14 \\
& Total insulin requirement & $0.84 \pm 0.25$ & $0.68 \pm 0.15$ & $0.93 \pm 0.21$ & $0.76 \pm 0.32$ & 0.03 \\
\hline
\end{tabular}

insulin analogues. Participants showed a better metabolic control as compared to the previous studies. Despite that, the incidence of hypoglycemic episodes was similar to that reported in other studies. ${ }^{7,8,17}$ No severe episode of hypoglycemia occurred during the camp, while in previous studies based on camps of longer duration severe episodes were registered. ${ }^{7}$

In agreement with other studies, ${ }^{9}$ initial doses of basal insulin were not reduced in all participants. The long half-life of IDeg can in theory represent an obstacle to a flexible management of insulin doses during irregular or short periods of physical activity. Our experience shows that an individualized approach based on $\mathrm{CHO}$ counting and ISF makes it possible to adjust IDeg doses, without increasing the risk of hypoglycemia while achieving a good metabolic control.

According to ISPAD recommendations, total insulin doses should be decreased by $20-25 \%$ the first day of the camp. ${ }^{5}$ However, a uniform approach could determine marked hyperglycemia during the first and second day of the camp in some participants. Furthermore, sedentary individuals would require a greater reduction of insulin doses as a consequence of the increase in physical activity. In our study, a decrease in total insulin doses was needed in $55 \%$ of the children, while an increase in total insulin doses was applied to $45 \%$ of them. The increase in total insulin doses in some patients is related to sub-optimal insulin treatment before the camp, mainly related to therapeutic inertia or fear of hypoglycemia. ${ }^{22}$

\section{Implications for clinical practice}

We documented a higher rate of hypoglycemia during the third day of the camp in all treatment groups, likely related to a stable, marked increase in insulin sensitivity. ${ }^{23}$ Therefore, in case of camps lasting longer than 3-4 days, it is plausible that a further reduction of basal insulin would be requested in all treatment schemes, along with a re- evaluation of I:CHO and ISF. Specific algorithms should be developed also for IDeg and timing of its application should be adapted to pharmacokinetics properties (e.g. IDeg might be reduced at the second day of the camp, while IGlar dose should be reduced at the third day). During therapy adjustments, even $\mathrm{CHO}$ counting, and dosage of short-acting insulin should require additional attention to minimize glycemic variability. Any possible advancement in knowledge and clinical recommendations regarding the management of children with T1DM attending a camp should underline the importance of a personalized approach in the therapy adjustment.

\section{Strengths and limitations}

Among the strengths, this is the first study assessing the impact of IDeg in a camp. Among the limitations, the absence of randomization does not allow an unbiased comparison among the three groups; furthermore, the small sample size does not allow the identification of possible statistically significant differences. For these reasons, the study has a descriptive nature.

\section{Conclusions}

In conclusion, the study shows similar effectiveness and safety of different insulin schemes during a pediatric summer diabetes camp when associated with a flexible algorithm for the management of T1DM based on CHO counting and ISF. Despite its longer half-life, IDeg use is not associated with an increased risk of hypoglycemia. Other studies are needed to further optimize insulin management during diabetes camps, particularly with reference to the last generation of short-acting and long-acting insulins. The role of continuous glucose monitoring systems in personalizing insulin treatment also deserves further investigation.

\section{References}

1. SJ B. Diabetes camping and youth support programs. In: Dekker M, editor. Paediatric Endocrinology: A Clinical Guide. New York; 1996. p. 671-6.

2. Phelan H, Lange K, Cengiz E, et al. ISPAD Clinical Practice Consensus Guidelines 2018: Diabetes education in children and adolescents. Pediatr Diabetes 2018;19:75-83.

3. Pihoker C, Forsander G, Fantahun B, et al. ISPAD Clinical Practice Consensus Guidelines 2018: The delivery of ambulatory diabetes care to children and adolescents with diabetes. Pediatr Diabetes 2018;19:84-104.

4. American Diabetes Association AD, Wendt LFC. Diabetes management at camps for children with diabetes. Diabetes Care 2012;35:S72-5.

5. Adolfsson P, Riddell MC, Taplin CE, et al. ISPAD Clinical Practice Consensus Guidelines 2018: Exercise in children and adolescents with diabetes. Pediatr Diabetes 2018;19:205-26.

6. Riddell MC, Gallen IW, Smart CE, et al. Exercise management in type 1 diabetes: a consensus statement. Lancet Diabetes Endocrinol 2017;5:377-90.

7. Gunasekera H, Ambler G. Safety and efficacy of blood glucose management practices at a diabetes camp. J Paediatr Child Health 2006;42:643-8.

8. Braatvedt GD, Mildenhall L, Patten C, Harris G. Insulin Requirements and Metabolic Control in Children with Diabetes Mellitus Attending a Summer Camp. Diabet Med 1997;14:258-61.

9. Miller AR, Nebesio TD, DiMeglio LA. Insulin dose changes in children attending a residential diabetes camp. Diabet Med 2011;28:480-6.

10. Bode BW, Buse JB, Fisher M, et al. Insulin degludec improves glycaemic control with lower nocturnal hypoglycaemia risk than insulin glargine in basal-bolus treatment with mealtime insulin aspart in Type 1 diabetes (BEGIN ${ }^{\circledR}$ Basal-Bolus Type 1): 2-year results of a randomized clinical trial. Diabet Med 2013;30:1293-7.

11. Heise T, Hermanski L, Nosek L, et al. Insulin degludec: four times lower pharmacodynamic variability than insulin glargine under steady-state conditions in type 1 diabetes. Diabetes Obes Metab 2012;14:859-64.

12. Moser O, Eckstein ML, Mueller A, et al. Reduction in insulin degludec dosing for multiple exercise sessions improves 
time spent in euglycaemia in people with type 1 diabetes: a randomised cross-over trial. Diabetes, Obes Metab. Wiley/Blackwell 2018 Oct 14

13. Moser O, Tschakert G, Mueller A, et al. Effects of High-Intensity Interval Exercise versus Moderate Continuous Exercise on Glucose Homeostasis and Hormone Response in Patients with Type 1 Diabetes Mellitus Using Novel Ultra-Long-Acting Insulin. Catapano A, editor. PLoS One 2015;10:e0136489.

14. Hopkins D, Lawrence IAN, Mansell P, et al. Improved biomedical and psychological outcomes 1 year after structured education in flexible insulin therapy for people with type 1 diabetes the U.K. DAFNE experience. Diabetes Care 2012;35:1638-42.

15. Abraham MB, Jones TW, Naranjo D, et al. ISPAD Clinical Practice Consensus Guidelines 2018: Assessment and man- agement of hypoglycemia in children and adolescents with diabetes. Pediatr Diabetes 2018;19:178-92.

16. Monnier L, Colette C, Wojtusciszyn A, et al. Toward Defining the Threshold Between Low and High Glucose Variability in Diabetes. Diabetes Care 2017;40:832-8.

17. Semiz S, Bilgin UO, Bundak R, Bircan I. Summer camps for diabetic children: an experience in Antalya, Turkey. Acta Diabetol 2000;37:197-200.

18. Misuraca A, Di Gennaro M, Lioniello $M$, et al. Summer camps for diabetic children: an experience in Campania, Italy. Diabetes Res Clin Pract 1996;32:91-6.

19. Hunter HL, Rosnov DL, Koontz D, Roberts MC. Camping Programs for Children with Chronic Illness as a Modality for Recreation, Treatment, and Evaluation: An Example of a
Mission-Based Program Evaluation of a Diabetes Camp. J Clin Psychol Med Settings 2006;13:64-77.

20. Karagüzel G, Bircan I, Erişir S, Bundak R. Metabolic control and educational status in children with type 1 diabetes: Effects of a summer camp and intensive insulin treatment. Acta Diabetol 2005;42:156-61.

21. Maslow GR, Lobato D. Diabetes summer camps: History, safety, and outcomes. Pediatr Diabetes. John Wiley \& Sons, Ltd. 2009;10:278-88.

22. Gonder-Frederick L, Nyer M, Shepard JA, et al. Assessing fear of hypoglycemia in children with Type 1 diabetes and their parents. Diabetes Manag. NIH Public Access 2011;1:62739.

23. Hawley JA, Lessard SJ. Exercise training-induced improvements in insulin action. Acta Physiol 2008;192:127-35. 